\title{
Field Trial on 5G New Radio Over Satellite
}

\author{
Florian Völk*, Robert T. Schwarz and Andreas Knopp \\ Chair of Signal Processing, Bundeswehr University Munich, Neubiberg, Germany
}

$5 G$ New Radio (NR) is the 3rd Generation Partnership Project (3GPP) radio access technology for the next generation mobile communications network. A major evolution of $5 \mathrm{G}$ constitutes the integration of non-terrestrial networks including geostationary and low Earth orbit satellites. The seamless integration of satellites in the terrestrial mobile network requires significant adaptations within the radio access network and the development of new features in the core network to cope with the specific satellite channel characteristics. To date, the $5 \mathrm{G}$ control and data plane has been standardized to handle only continuous backhaul communication between the network components. However, a mobile satellite enabled next generation Node B (gNB) located in a vehicle or in a moving aerial platform needs to be able to handle frequent backhaul outages of various duration as well as longer signal delays as opposed to short terrestrial connections via

OPEN ACCESS

Edited by:

Carlos Mosquera, University of Vigo, Spain

Reviewed by:

Wael Jaafar,

Carleton University, Canada Pantelis-Daniel Arapoglou, European Space Research and Technology Centre (ESTEC),

Netherlands

*Correspondence: Florian Völk papers.sp@unibw.de

Specialty section: This article was submitted to Aerial and Space Networks,

a section of the journal Frontiers in Communications and Networks

Received: 27 February 2021 Accepted: 31 May 2021 Published: 18 June 2021

Citation:

Völk F, Schwarz RT and Knopp A (2021) Field Trial on 5G New Radio

Over Satellite.

Front. Comms. Net 2:673534. doi: 10.3389/frcmn.2021.673534 fiber. In this paper, we report the results of an over-the-air (OTA) field trial comprising a mobile edge node connected to the $5 \mathrm{G}$ standalone core network components over a geostationary satellite. We analyze Transmission Control Protocol (TCP) acceleration and GPRS Tunneling Protocol (GTP)/TCP/Internet Protocol (IP) header compression features through the GTP. Moreover, the influence of short and long interruptions in the communication between the edge node and the central components on the entire system performance is investigated. The header compression and TCP acceleration modules were implemented on the satellite modems and are now part of the protocol stack of these devices. The results show up to $12 \%$ higher data rates for the $5 \mathrm{G}$ user equipment (UE), on a $1.5 \mathrm{MHz}$ single carrier return link compared to deactivated TCP acceleration and header compression. We increased the data rate by $20 \%$ on the $4.5 \mathrm{MHz}$ DVB-S2X forward link between the UE and 5G core. Moreover, our measurements reveal that even satellite-enabled gNB mobility is possible with the current Release 15 standard. After a short outage of the satellite connection due to shadowing, the UE can successfully re-establish the user plane connection to the core network. Our results will facilitate the full integration of satellite components in $5 \mathrm{G}$ through open and standard solutions.

Keywords: 5G new radio, non-terrestrial networks, geostationary satellite, field trial, satellite backhauling

\section{INTRODUCTION}

The next generation mobile communications standard $5 \mathrm{G}$ will decisively improve the possibilities for real-time communication. With a significantly higher data rate and very short response times compared to previous standards, 5G will tackle the current requirements for communication in a fully connected society much more comprehensively than has been possible to date. The specification 
of $5 \mathrm{G}$ creates a crucial foundation that allows integration of new applications into intelligent mobility and the Internet of Things (IoT). Europe, with industries and institutions such as the European Space Agency (ESA) is playing an active role in the definition and development of new releases to secure long-term innovation leadership in this key technology field.

The deployment of $5 \mathrm{G}$ Release 15 networks by terrestrial mobile network operators (MNOs) has already begun and delivers enhanced mobile broadband (eMBB) services in small cells. The integration of satellite components, so-called NonTerrestrial Networks (NTNs), in terrestrial infrastructure is currently studied and will be specified in Release 17. The widespread use of communication satellites in the future network-of-networks is envisaged by 2023. An exemplary use case of satellites in $5 \mathrm{G}$ is backhauling of cell traffic to connect the MNO core network with the edges. In current terrestrial deployments, this backhaul is based on fiber connections or microwave links. Thus, the control plane in Release 15 relies on continuous backhaul connections between the network components. However, a next generation Node B backhauled via satellite has to handle longer signal delays as opposed to short terrestrial connections over fiber. If the gNB itself is mobile, link outages of varying duration need to be considered for the satellite backhaul link.

Several testbeds have been developed in recent years to support the integration of NTNs. The concept of testbeds and open research infrastructures play a vital role in the development of future releases. As the traditional borders of network and service layers are vanishing, cross-layer experimental platforms are necessary and allow testing and validation of innovative ideas, rapid prototyping, and they generally support migration activities. Running testbeds helps finding compatibility issues, bottlenecks and protocol inconsistencies between the different 5G and satellite components. As part of the Satellite and Terrestrial Network for 5G (SaT5G) project, satellite-based backhaul and traffic offloading solutions have been investigated under real conditions, Ge et al. (2019); Liolis et al. (2018); Goratti et al. (2020); Evans et al. (2021). Another example is the 5G Vertical Innovation Infrastructure (5G-VINNI) project developing a $5 \mathrm{G}$ facility that will demonstrate satellite based $5 \mathrm{G}$ implementations, and allow vertical industries to test and validate specific 5G applications over satellite, Politis et al. (2019), Politis et al. (2021). In this paper, we report the results of an OTA field trial that we ran as part of the ESA ARTES Advanced Technology project Demonstrator for Satellite-Terrestrial Integration in the 5G Context (SATis5), SATis5 (2020). In SATis5, we developed a satellite-terrestrial Proof of Concept $(\mathrm{PoC})$ testbed to validate the use of satellite as a mean of connectivity in NTN use cases, providing first hand results and insight into what satellite and terrestrial network convergence can offer.

The Transmission Control Protocol is the most important transport protocol on the Internet. TCP is used as the transport layer protocol by many Internet Protocol based applications over satellite. However, it is well-known that TCP performance is limited over satellite links. The performace is limited due to the long propagation delays and the congestion control features together with the slow-start mechanism of TCP, Allman et al.
(2020) (accessed December 2, 2020). Therefore, several extensions to TCP have been developed that are known as "TCP acceleration features" and which are usually implemented in the satellite modem as the interface between the satellite and terrestrial network. One method is known as Split-TCP, in which two Performance Enhancing Proxys (PEPs) encapsulate the satellite link and remove TCP from the satellite link. TCP spoofing is the PEP technique that imitates a terrestrial TCP session by sending false TCP packet acknowledgments to the sender. However, these enhancements cannot be applied straightforward in $5 \mathrm{G}$ satellite backhauling because the TCP traffic is encapsulated in a GPRS Tunneling Protocol. Compared to $4 \mathrm{G}$ there are some adjustments in the 5G GTP protocol header such as the activated Next Extension Headers. Most of the commercial off-the-shelf (COTS) gNB rely on this header format that could be problematic for GTP acceleration utilizing legacy satellite modems.

In this context, the satellite modem is a key component to realize a seamless integration of NTNs in $5 \mathrm{G}$ Release 17 . The software of current state-of-the-art satellite modems must be adapted to allow TCP acceleration, but also other bandwidth enhancing features such as protocol header compression. As part of SATis5, the protocol stack of the satellite modems was enhanced by our project partner. We tested and analyzed the latest ST Engineering iDirect software release of TCP acceleration and header compression of various protocols through 5G GTP tunnels. The OTA test setup comprises a mobile edge node that is connected to the $5 \mathrm{G}$ standalone core network components over a geostationary satellite backhaul.

\section{USE CASE: EMERGENCY SERVICES}

SATis5 analyses, besides many other use cases, a mobile 5G network that enables the establishment of local networks for emergency service use cases. A delay in setup of a communications infrastructure for emergency services could potentially make the difference between life and death.

Using the same slices for video acquisition, video delivery and IoT, the SATis5 5G nomadic network is able to provide comprehensive use cases addressing the different operational requirements with local command centers and with central node sites. Additional use cases can be dynamically deployed networks for short duration of time such as live TV video transmissions, logistics of public events (e.g. tours of concerts), or construction sites. In many use cases, the end device for IPbased services is often not equipped with its own $5 \mathrm{G}$ modem. For cost or space reasons, Wi-Fi is used instead. In many cases, a 5Gbased Wi-Fi access point can be the optimal solution to enable the integration of as many devices as needed.

One possible example is the design and operation of a deployable, portable, and temporary hospital in Figure 1. Most of the required medical IoT devices need a data connection, but at the same time, a flexible relocation of these devices should be possible. Wi-Fi alone is not sufficient for connecting to the central network due to the limited signal strength and cables preventing the fastest possible 


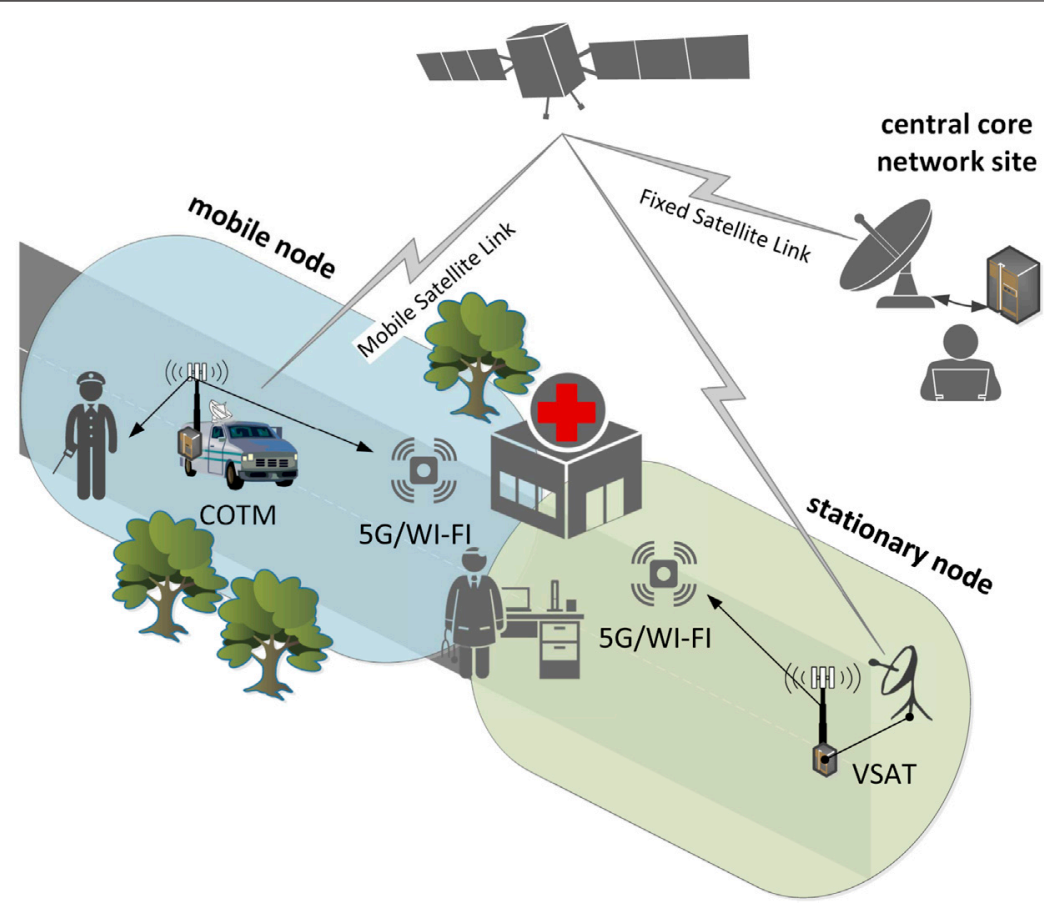

FIGURE 1 | Remote hospital deployment.

commissioning. The portable hospital should be operational anywhere, which is why connecting to the central network via satellite is the most effective method. By using a $5 \mathrm{G}$ mobile base station connected via satellite, large-scale coverage can be ensured very quickly in the area of operation. The local connection of the individual IoT devices takes place either via Ethernet cable or via $\mathrm{Wi}$-Fi using 5G-based routers as relay nodes since most medical devices do not have their own $5 \mathrm{G}$ modem. Either Wi-Fi or Ethernet is usually available. The combination of wireless local area networks and satellite based wide area networks is a great choice for many scenarios. The main advantages are:

- Cheap Wi-Fi chips with very small integrated antennas at the user equipment UE.

- Increased cell coverage due to high gain antennas at the 5G/ Wi-Fi relay nodes.

- Much higher transmit power of the gNB compared to Wi-Fi Access points.

\section{TESTBED DEMONSTRATION SETUP}

The existing SATis5 testbed, Corici et al. (2020), was updated with an additional central node in Munich, Germany (see Figure 2). A gNB backhauled via satellite was operated in 5G standalone mode. This enables the end-to-end operation of a COTS 5G UE over satellite. The extension allows the first demonstration of backhauling using a third party gNB and a COTS 5G UE. The extension of the Munich SATis5 testbed brings some advantages compared to the initial distributed testbed. There is no need for an additional internet connection to connect the satellite hub equipment to the core network. Thus, the end-to-end delay can be reduced. In addition, the entire lab can be configured through a single virtual private network (VPN) connection. This allows third parties to operate the Munich SATis5 testbed extension remotely for vertical use case pilots.

Here we describe the design of our satellite communication (SATCOM) enabled 5G testbed as illustrated in Figure 2. The SATis5 Munich testbed setup consisted of a Huawei CPE 5G router, which was connected to the Amarisoft $5 \mathrm{G}$ base station. We used a $40 \mathrm{MHz}$ TDD carrier for the terrestrial $5 \mathrm{G}$ radio network. ETTUS B210 USRP provided the RF Frontend for the gNB. We implemented the use case as part of the mobile edge node, which was connected to the main core network computer (i7-8700 CPU @ $3.20 \mathrm{GHz}, 32 \mathrm{~GB}$ RAM). Fraunhofer FOKUS provided the $5 \mathrm{G}$ standalone core network based on the latest 3rd Generation Partnership Project Release 15. The mobile edge node, a vehicle with energy storage, provided the $5 \mathrm{G}$ cell backhauled via satellite. The $5 \mathrm{G}$ base station software, which was running on a consumer computer (i7-9850H CPU @ 2.60 GHz, 16 GB RAM) together with the edge node software, was connected to the ST Engineering iDirect MDM 5000 satellite modem that established a satellite connection to the Munich satellite hub station. Modem and base station were installed in the vehicle to communicate via the Communication on-the-Move (COTM) antenna, installed on top of the vehicle, with the satellite. We used a Raspberry Pi Zero with ublox LARA-R211 LTE CAT1 modem to connect either via Wi-Fi to the $5 \mathrm{G} \mathrm{UE}$ or via $4 \mathrm{G}$ 


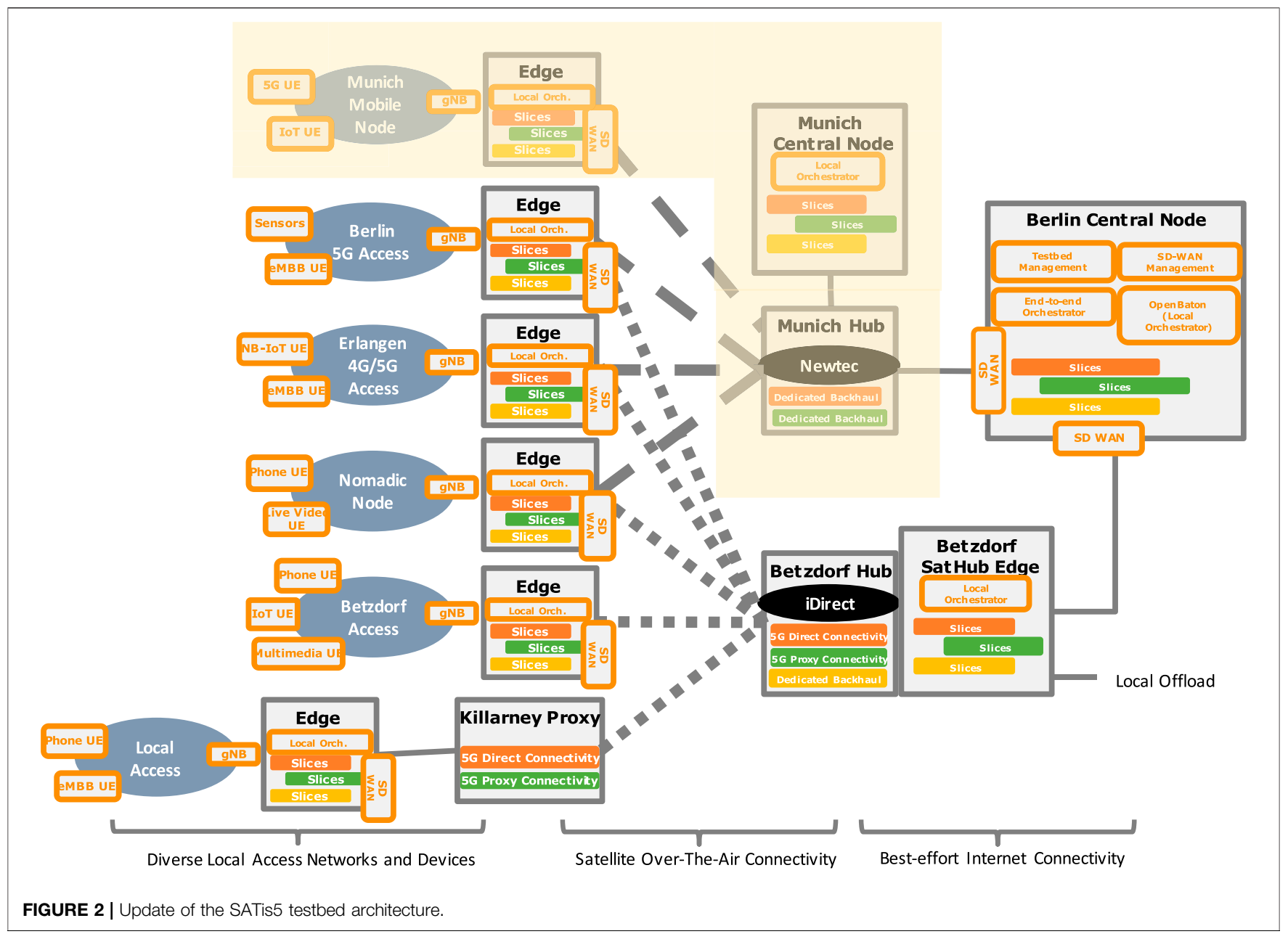

connection to the mobile edge node. Furthermore, we installed an additional EPC at the core computer to enable the edge node to provide either a $4 \mathrm{G}$ or a $5 \mathrm{G}$ cell. However, the main scenario in this study was based on the $5 \mathrm{G}$ standalone architecture.

An end-to-end field trial was performed over a transparent geostationary satellite. SES's ASTRA 2F satellite provided seamless connectivity between the mobile edge node and the ST Engineering iDirect Dialog 1IF satellite hub station located at the UniBw teleport in Munich, Germany. The satellite hub station has been directly connected to the $5 \mathrm{G}$ core network nearby as shown in Figure 3. ST Engineering iDirects TelliNet is a distributed PEP running on the satellite modems and the Hub for acceleration of TCP, HTTP, SMB and POP3 protocols via satellite. Besides this, it provides general payload and header compression and encryption. In order to validate the high frequency performance of the COTM antenna and the ST Engineering iDirect MDM 5000 satellite modem under typical operational conditions, we established a bi-directional satellite connection with forward and return links between the COTM antenna and the hub station in the Ku-band. The bandwidth allocated for the DVB-S2X satellite forward link was $4.5 \mathrm{MHz}$ and for the single carrier return link 1.5 $\mathrm{MHz}$. The satellite backhaul link between the $5 \mathrm{G}$ Core to the gNB is called forward link and does not include the downlink between the gNB to a UE. The return link is between the gNB to the $5 \mathrm{G}$ Core not including the UE uplink to the gNB. Compared to the terrestrial link between gNB and the UE with a $40 \mathrm{MHz} 5 \mathrm{G}$ TDD carrier, the satellite backhaul network constitutes the bottleneck in terms of peak data rates. However, even in terrestrial networks a overbooking of the transport network is considered by the MNO, which leads to packet loss. This additional packet loss is reducing TCP data sent via the UE toward the base station.

The hub station was a $7.6 \mathrm{~m}$ anchor station equipped with a $400 \mathrm{~W}$ high power amplifier resulting in an equivalent isotropically radiated power (EIRP) of approximately $85 \mathrm{dBW}$ in saturation and a $\mathrm{G} / \mathrm{T}$ of $37.7 \mathrm{~dB} / \mathrm{K}$. The $0.46 \mathrm{~m}$ COTM antenna provides a maximum EIRP of $44.8 \mathrm{dBW}$ and a $\mathrm{G} / \mathrm{T}$ of $12.4 \mathrm{~dB} / \mathrm{K}$. A link-budget calculation for the SATCOM links is provided in Table 1. All components of the testbed can also be accessed externally via a VPN or remote monitoring applications and can be configured accordingly for the use case scenario. In addition, we used the iPerf3 network tool running 


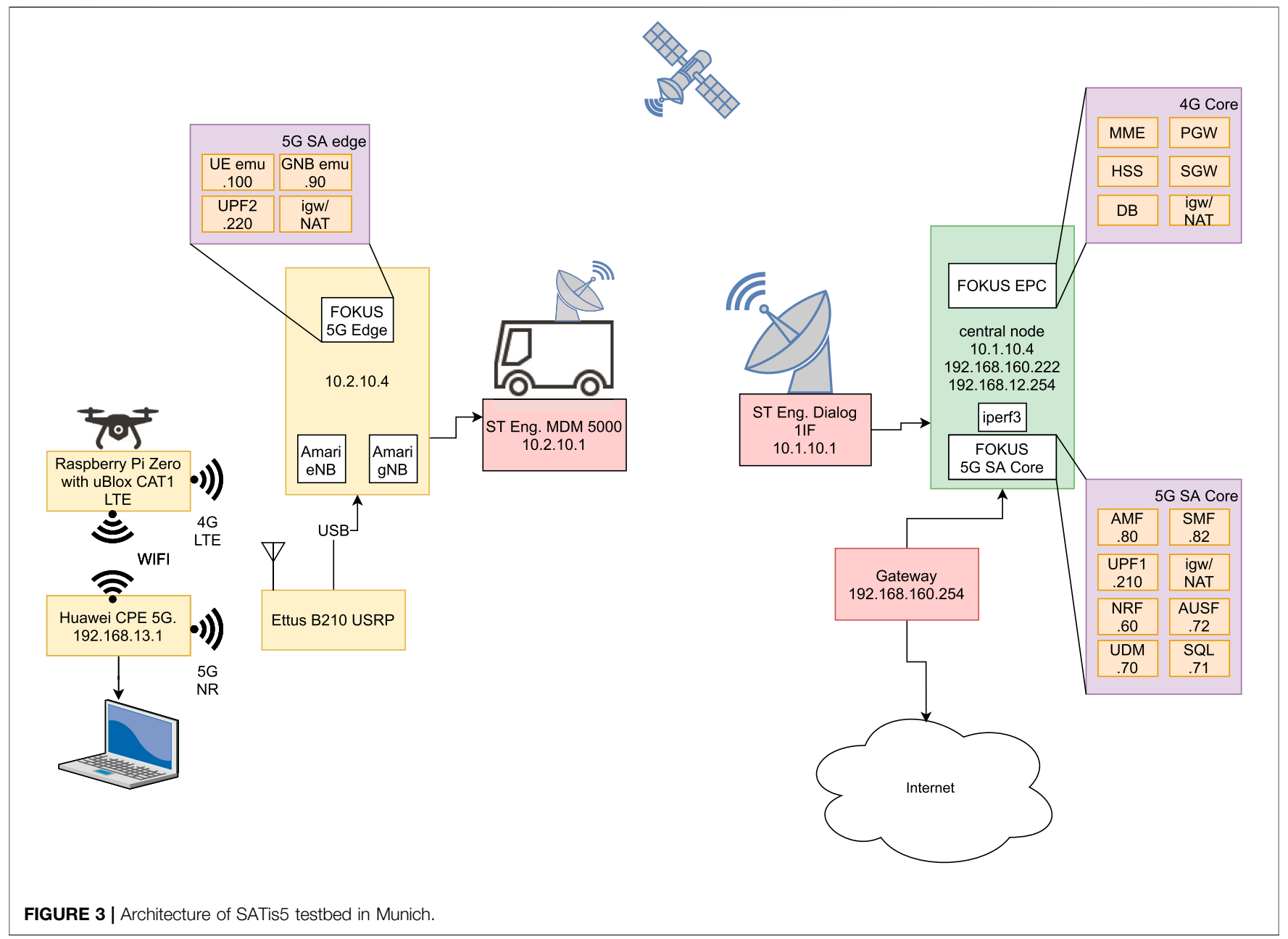

TABLE 1 | Link-budget calculation.

\begin{tabular}{llcc} 
Parameter & Unit & Return link & Forward link \\
\hline Symbol rate & $\mathrm{MS} / \mathrm{s}$ & 1.25 & 3.75 \\
Roll-off factor & & 0.05 & 0.20 \\
Allocated bandwidth & $\mathrm{MHz}$ & 1.31 & 4.50 \\
Tx antenna diameter & $\mathrm{m}$ & 0.46 & 7.60 \\
Total EIRP required & $\mathrm{dBW}$ & 39.90 & 57.47 \\
Distance to satellite & $\mathrm{km}$ & 38,462 & 38,462 \\
FSPL & $\mathrm{dB}$ & 207 & 207 \\
Attmospheric Att & $\mathrm{dB}$ & 0.35 & 0.35 \\
Transponder beam-peak G/T & $\mathrm{dB} / \mathrm{K}$ & 12.97 & 12.97 \\
Rx antenna diameter & $\mathrm{m}$ & 7.60 & 0.46 \\
Effective G/T Rx antenna & $\mathrm{dB} / \mathrm{K}$ & 35.95 & 11.56 \\
\hline Total C/(N + I) clear sky & $\mathrm{dB}$ & 2.37 & 3.08 \\
Total Eb/no clear sky & $\mathrm{dB}$ & 2.83 & 3.23 \\
Expected data rate & $\mathrm{Mb} / \mathrm{s}$ & 1.81 & 6.00
\end{tabular}

on the 5G Core computer to measure TCP and User Datagram Protocol (UDP) bandwidth performance between UEs and the $5 \mathrm{G}$ Core. iPerf3 is a command line program that accepts a number of different options allowing insights into network availability, bandwidth, jitter and potential packet loss.

\section{RESULTS}

\subsection{GPRS Tunneling Protocol / TCP Acceleration and Header Compression}

GTP is used as a protocol to carry user and control data in $5 \mathrm{G}$ between the core network and the radio access network (RAN). In case of user data, the protocol is called GPRS Tunneling ProtocolU (GTP-U). For a single TCP connection between two UEs, two GTP-U tunnels are established: One uplink GTP-U tunnel between the UE via the RAN to the core network, and one downlink GTP-U tunnel in the opposite direction. The GTP-U data packet comprises five headers (see Figure 4). Two of them are IP headers (an inner and an outer IP header) with a standard header size of 20 bytes. The GTP-U tunnel is based on the UDP/ IP protocol stack and UDP ( 8 bytes) and IP headers ( 20 bytes) are added before the GTP-U header. A standard satellite header compression function is able to reduce the header sizes of the outer protocols (IP and UDP), but it fails to reduce the inner protocol header sizes (IP and TCP or UDP) as they are part of the GTP-U header.

After receiving the IP packet from the Huawei 5G UE, the Amarisoft gNB adds a GTP tunnel header, consisting of the 


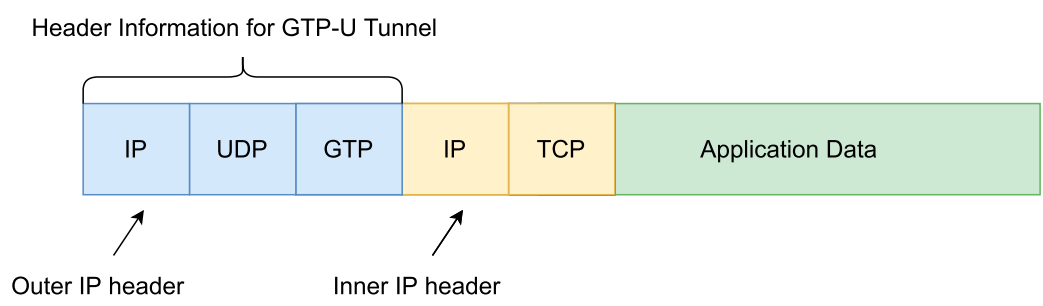

FIGURE 4 | Headers for data transfer across 5G GTP-U tunnel, Johnson (2019).
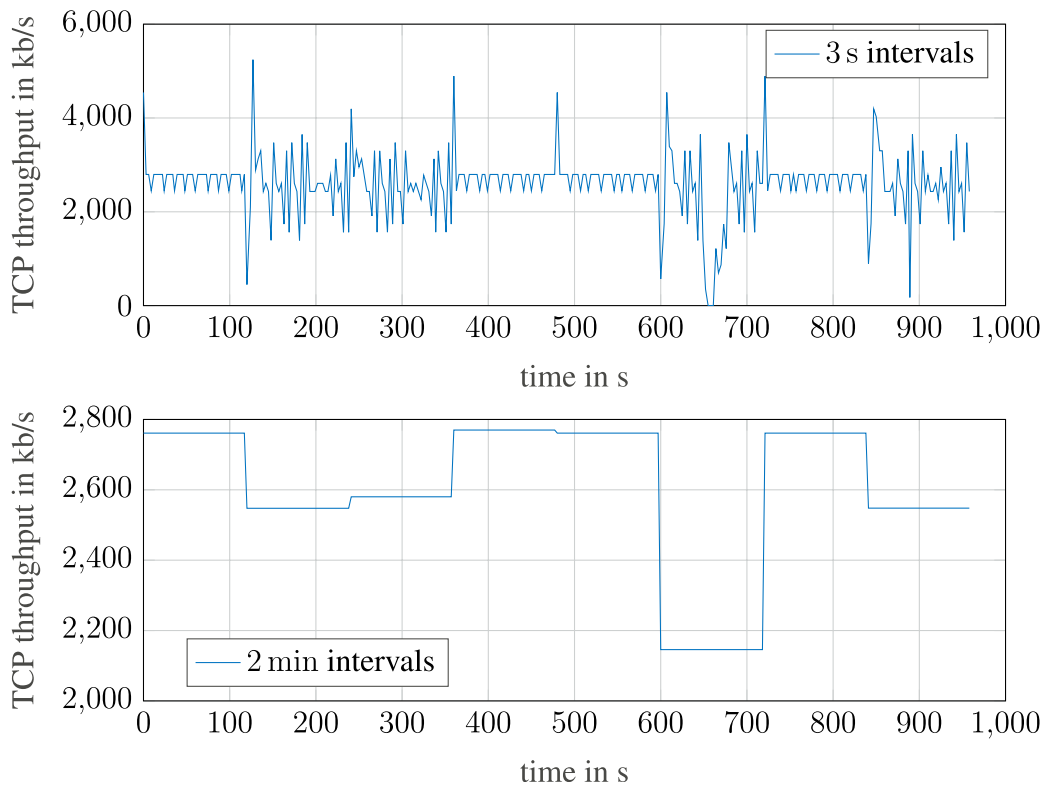

FIGURE 5 | TCP throughput measurements on the return link (static scenario).

three headers (see Figure 4 again). The gNB sends the IP packet to the user plane function (UPF) of the $5 \mathrm{G}$ Core. The UPF then removes all three headers (outer IP header/UDP header/GTP header) from the packet and forwards the original inner IP packet (from the $5 \mathrm{G}$ UE) to the desired IP network.

In this measurement, we investigated the functionality of TCP acceleration and header compression on the $5 \mathrm{G}$ end-toend application over satellite. To this end, the GTP-U tunnel was terminated at the satellite modem, and bi-directional TCP acceleration based on the TelliNet PEP application on the satellite modems in the vehicle and the hub were applied. Both satellite modems, in the hub and the vehicle, intercept the GTP-U tunnel transparently. The GTP-U tunnel was opened and the satellite modem detected the enclosed TCP sessions. The satellite modem removed the TCP encapsulation from the sender and transmitted the payload data through the satellite connection to the receiver where the receiving satellite modem re-encapsulated the data. In addition, header compression was enabled to reduce the number of bytes on the satellite link.

We used the iperf3 application software to measure the endto-end TCP throughput in both direction (forward and return). The total measurement time was 16 min for each direction, which we split into eight consecutive 2 min time slots. In four of the eight 2 min slots, the TCP acceleration and header compression features were enabled, and in the other four time slots these features were disabled.

The results for forward and return direction are shown in Figures 5, 6, respectively. iperf3 was configured to report the measured data rates in $3 \mathrm{~s}$ time intervals (upper part of each Figure) and in 2 min time intervals (lower part of the Figures). On the return link (Figure 5), TCP and header compression features were activated between 0s120s, 360s600s, and 720s840s. On the forward link (Figure 6), TCP and header compression features were activated between 120s240s, 360s600s, and 840s960s. The curves show that, with activated TCP acceleration, the slow-start phase of TCP is effectively avoided. Moreover, the data rate is 

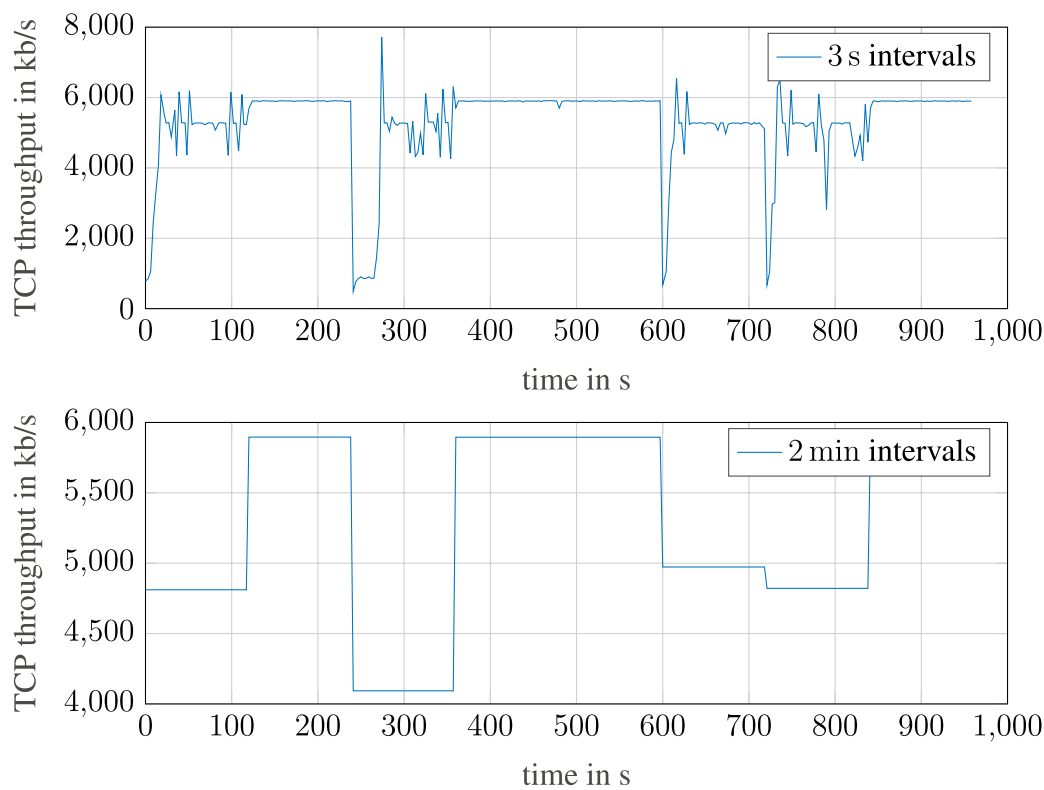

FIGURE 6 | TCP throughput measurements on the forward link (static scenario).

\begin{tabular}{|c|c|c|c|c|c|c|c|c|c|}
\hline \multirow{4}{*}{$\begin{array}{c}20 \\
\text { Bytes } \\
\text { Header } \\
\text { Size }\end{array}$} & Version & PT & 0 & $E$ & S & PN & Message Type & & ngth \\
\hline & \multicolumn{9}{|c|}{ Tunnel Endpoint Identifier } \\
\hline & \multicolumn{7}{|c|}{ Sequence Number } & N-PDU Number & Next Extension Header Type \\
\hline & \multicolumn{5}{|c|}{ Extension Header Length } & \multicolumn{4}{|c|}{ PDU Session Container } \\
\hline
\end{tabular}

FIGURE 7 | GTP-U header, Johnson (2019).

more stable. With enabled TCP acceleration and header compression features, the TCP data rate could be improved by 12 and $20 \%$ for the return and forward link, respectively.

During the field trials we were also able to identify incompatibilities between different protocol implementations in the satellite modems and the gNB and UE. For example, GTP Next Extension Header is currently not supported by the satellite modems of the testbed. Figure 7 shows the GTP-U header which is specified within 3GPP TS 29.281. GTP-U headers have a minimum length of 8 bytes and three header flags (E, S, and PN) to highlight the presence of additional optional fields. If the GTP Next Extension Header flag had been set, the modems were not able to terminate properly the GTP tunnels and perform TCP acceleration and header compression to the inner header. GTP Next Extension Headers have been rarely used in $4 \mathrm{G}$ deployments, but the modems should be updated now to be fully comply with future $5 \mathrm{G}$ equipment because many base station manufacturers tend to rely on this feature.

\subsection{Mobile Backhauling}

We investigated how the mobility of the mobile edge node affects the mobile radio connection of the satellite enabled $5 \mathrm{G}$ cell within the vehicle. For this purpose, we used a test site in which the vehicle has line of sight to the satellite in most cases. The test track includes some directional changes. In addition, trees in some places interrupt the line of sight for a few seconds. We investigated how the unstable satellite link affects the cellular $5 \mathrm{G}$ cell inside the vehicle. The aim of the investigation was to analyze the speed of the 5G User-Plane re-establishment after a satellite backhaul interruption.

The COTM antenna has several integrated tracking sensors for precise tracking of the satellite while driving. The sensors allow a short initial acquisition and immediate re-establishment of the communication link after shadowing by trees or street lamps.

For the validation and compatibility test between the UE, backhauled via satellite, and the 5G Core Internet Control Message Protocol (ICMP) messages were used. Therefore, we 


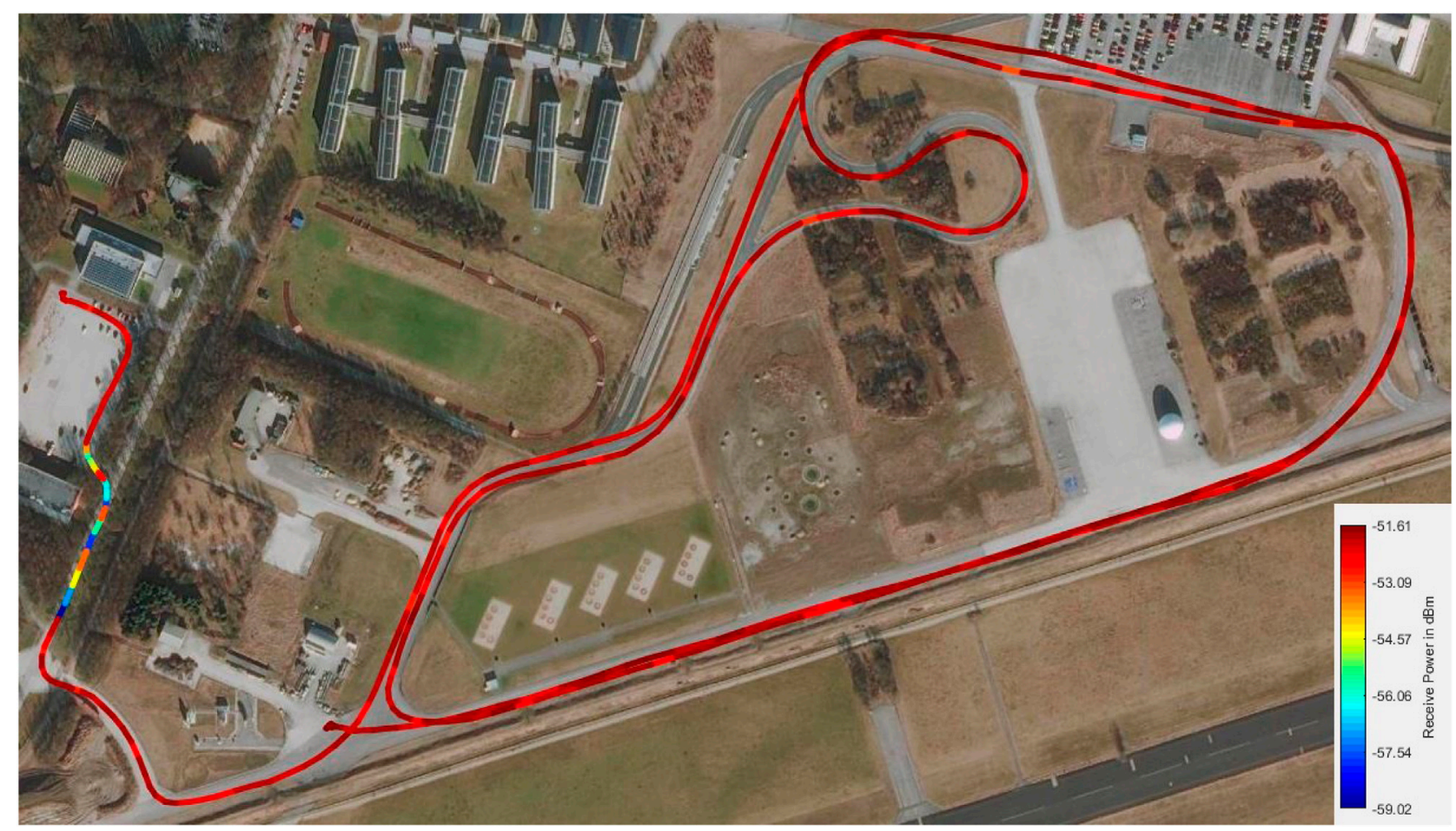

FIGURE 8 | Receive signal power in $\mathrm{dBm}$ at different locations of the test track.
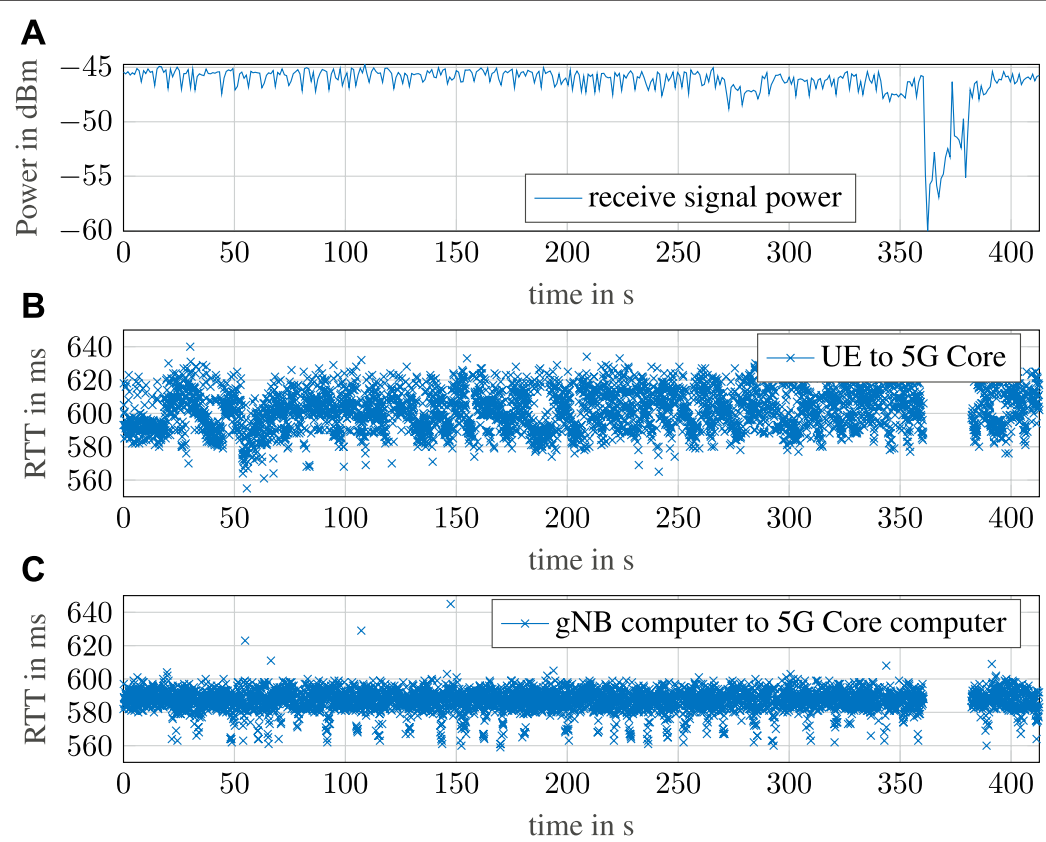

FIGURE 9| Measurement results showing the receive signal power (satellite modem) in $\mathrm{dBm}(\mathbf{A})$ and the round trip times (RTTs) between UE/gNB and $5 \mathrm{G}$ Core in both directions $\mathbf{( B , C )}$

sent ICMP messages encapsulated in the GTP-U tunnel from the UE to the $5 \mathrm{G}$ core network. Moreover, an analysis showed a second ICMP stream from the gNB computer to the core computer to analyze when the satellite backhaul is available. We sent ICMP messages every $0.1 \mathrm{~s}$. The average speed during the test drive was approximately $50 \mathrm{~km} / \mathrm{h}$. 

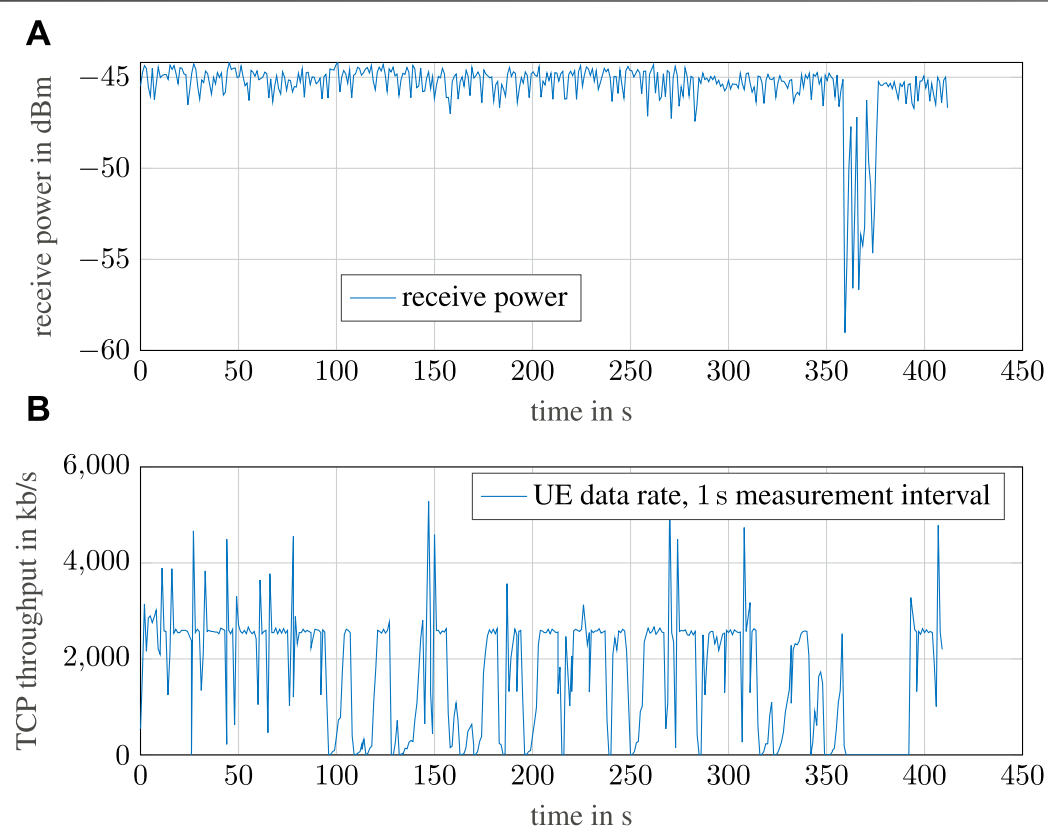

FIGURE 10| Measurement results showing the receive signal power (satellite modem) in $\mathrm{dBm}$ (A) and the TCP throughput measurements on the return link (mobile scenario) (B)

A test drive consisted of two laps on the test track, which we drove counter clockwise. The starting position was close to the antenna site in the southwest of the test track. During the first lap, we also drove the roundabout of the test site. The vehicle left the test site after two laps, and turned onto a road with many trees. After a few hundred meters, the vehicle left this road and turned into a parking lot. We stopped measurements at the end of the parking lot. Due to shadowing, the vehicle lost line of sight to the satellite for a few seconds. Moreover, a very short period of shadowing can be observed after the roundabout as illustrated in Figure 8.

As shown in Figure 9, both the ICMP Messages of the UE and the gNB computer recovered quickly after the shadowing period. It can be observed that during the measurement drive a constant ICMP stream was received at the core computer. However, the RTT of the UE is a little bit longer compared with the RTT of the gNB computer. Moreover, a larger variance of the UE RTT can be observed. This is mainly because of the additional radio interface between gNB and UE.

The measurement results in Figure 10 show that the TCP/IP data rate between the $5 \mathrm{G}$ UE and the $5 \mathrm{G}$ Core remained almost constant until the vehicle's direction of travel changed. With every further change in the direction, there is a brief drop in the data rate, which then rises again slowly to the maximum value. Supposedly, the drop in the data rate is due to bit errors caused by the Doppler shift. We observed the same behavior for a TCP/IP iperf measurement over the satellite link without using the $5 \mathrm{G}$ equipment. The effect of Doppler Shift is usually negligible for the fixed service application of geostationary satellites. The data rate increases slowly because no TCP acceleration was activated. Amarisoft's 5G base station is utilizing the GTP Next
Extension Headers which limits the application of TCP acceleration over the mobile satellite backhaul. However, the results show that even in a worst-case scenario, including shadowing and high bit errors due to the uncompensated Doppler shift, 5G communication via satellite is feasible. The unstable backhaul does not largely affect the $5 \mathrm{G}$ communication.

\section{FUTURE WORK}

In the last decade, SATCOM has enormously evolved. The technology progressed from broadcasting relays in space to high-capacity, stable, broadband IP access networks. Technical developments, such as the upcoming constellations of low Earth orbit satellites, are enabling space performance that was barely imaginable a few years ago. A major evolution of the $5 \mathrm{G}$ standard is the integration of NTN including both geostationary and low Earth orbit satellite constellations in 3GPP Release 17.

The evaluation of backhauling use cases via non-geostationary satellites is demonstrating latency close to the terrestrial networks. Starlink, a SpaceX company, is already providing a user terminal and access to their satellites during a beta test in North America and Europe. 5G backhauling tests with low-flying satellites could significantly boost the acceptance of space technologies for global $5 \mathrm{G}$ services.

Direct Access via satellite using a $5 \mathrm{G}$ UE is equally important as backhauling. UE and gNB prototype developments for satellite in parallel to the 3GPP standardization process is required to provide a test platform for research and industry. A software defined radio (SDR) system performs most of the signal processing using a general-purpose computer, combined with 
dedicated hardware such as signal processors and/or FPGAs if required. Receiver bandwidths of up to $160 \mathrm{MHz}$ can be realized with such systems. Both, the srsRAN and OpenAirInterface, Kaltenberger et al. (2020), open source projects are promising candidates for the SDR development of early $5 \mathrm{G}$ non-terrestrial networks.

\section{CONCLUSION}

In this article, we summarized the results of our recent OTA field trial that has been conducted as part of the ESA ARTES Advanced Technology project SATis5. We have shown that the integration of NTNs into 5G networks is feasible, even in a mobile satellite scenario. Key component is the interface between terrestrial and satellite network, and in this context special attention must be devoted to the satellite modem. A standard compliant GTP decapsulation is required to enable TCP acceleration in a $5 \mathrm{G}$ transport network between the access and the core network. The demonstration has revealed the difference of the GTP Next Extension Header between $5 \mathrm{G}$ and previous standard implementations, which highlighted the benefits of testbeds and open test infrastructures. Our test results illustrate the importance of considering all protocol layers in order to use the satellite backhaul as efficiently as possible. Mobile satellite backhauls address many of the requirements of verticals such as emergency services while minimal adaptations within the terrestrial 5G and COTM equipment are required for backhauling via satellite.

\section{REFERENCES}

Allman, M., Glover, D., and Sanchez, L. (2020). Enhancing TCP over Satellite Channels Using Standard Mechanisms. Available at: https://tools.ietf.org/html/ rfc2488.(.(accessed December 2, 2020).

Corici, M., Liolis, K., Politis, C., Geurtz, A., Cahill, J., Bunyan, S., et al. (2020). Satellite Is $5 G$ - SATis5 Whitepaper. Tech. rep.

Evans, B., Wang, N., Rahulan, Y., Kumar, S., Cahill, J., Kavanagh, M., et al. (2021). An Integrated Satellite-Terrestrial 5G Network and its Use to Demonstrate 5G Use Cases. Int. J. Satell Commun. Netw. doi:10.1002/sat.1393

Ge, C., Wang, N., Selinis, I., Cahill, J., Kavanagh, M., Liolis, K., et al. (2019). QoEAssured Live Streaming via Satellite Backhaul in 5G Networks. IEEE Trans. Broadcast. 65, 381-391. doi:10.1109/TBC.2019.2901397

Goratti, L., Herle, S., Betz, T., Temprado Garriga, E., Khalili, H., Khodashenas, P. S., et al. (2020). Satellite Integration into 5G: Accent on Testbed Implementation and Demonstration Results for $5 \mathrm{G}$ Aero Platform Backhauling Use Case. Int. J. Satell Commun. Netw. , 1-20. doi:10.1002/sat.1379

Johnson, C. (2019). 5G New Radio In Bullets - (Printed in the United States of America: Independently Published).

Kaltenberger, F., Silva, A. P., Gosain, A., Wang, L., and Nguyen, T.-T. (2020). OpenAirInterface: Democratizing Innovation in the $5 \mathrm{G}$ Era. Computer Networks 176, 107284. doi:10.1016/j.comnet.2020.107284

Liolis, K., Geurtz, A., Sperber, R., Schulz, D., Watts, S., Poziopoulou, G., et al. (2018). Use Cases and Scenarios of 5G Integrated Satellite-terrestrial Networks

\section{DATA AVAILABILITY STATEMENT}

The original contributions presented in the study are included in the article/Supplementary Material, further inquiries can be directed to the corresponding author.

\section{AUTHOR CONTRIBUTIONS}

FV and RS conducted the measurement campaign and analyzed the results. The measurements were performed by FV. AK was responsible for planning and execution of the research activities.

\section{FUNDING}

The work presented is part of the ESA ARTES Advanced Technology project "SATis5: Demonstrator for Satellite Terrestrial Integration in the 5G Context," ESA Contract No. $4000120663 / 17 / \mathrm{NL} / \mathrm{CLP}$. The views expressed herein can in no way be taken to reflect the official opinion of the ESA.

\section{ACKNOWLEDGMENTS}

We would like to thank our partners Fraunhofer FOKUS and ST Engineering iDirect for their support to setup the Munich SATis5 testbed site. We also thank SES and the 5G-VINNI project consortium for providing us with satellite capacity.

for Enhanced mobile Broadband: The SaT5G Approach. Int. J. Satell Commun. Netw. 37, 91-112. doi:10.1002/sat.1245

Politis, C., Liolis, K., Corici, M., Troudt, E., Szabo, Z., and Cahill, J. (2019). Design of Moving Experimentation Facility to Showcase Satellite Integration into 5G. 2019 European Conference on Networks and Communications (EuCNC); 1821 June 2019; Valencia, Spain, IEEE, 177-181. doi:10.1109/ EuCNC.2019.8801980

Politis, C., Liolis, K., Grønsund, P., and Heck, S. (2021). Use Cases and Testbed Solutions for $5 \mathrm{G}$ Cellular Backhauling via Satellite. Int. J. Satell Commun. Netw. doi:10.1002/sat.1395

SATis5 (2020). Demonstrator for Satellite-Terrestrial Integration in the 5G Context. Available at: https://satis5.eurescom.eu/ (Accessed 02 25, 2021).

Conflict of Interest: The authors declare that the research was conducted in the absence of any commercial or financial relationships that could be construed as a potential conflict of interest.

Copyright (c) 2021 Völk, Schwarz and Knopp. This is an open-access article distributed under the terms of the Creative Commons Attribution License (CC $B Y)$. The use, distribution or reproduction in other forums is permitted, provided the original author(s) and the copyright owner(s) are credited and that the original publication in this journal is cited, in accordance with accepted academic practice. No use, distribution or reproduction is permitted which does not comply with these terms. 